\title{
Emerging evidence of the association between cognitive deficits and arm motor recovery after stroke: A meta-analysis
}

\author{
Aditi A. Mullick ${ }^{\mathrm{a}, \mathrm{b}}$, Sandeep K. Subramanian ${ }^{\mathrm{b}, \mathrm{c}}$ and Mindy F. Levin ${ }^{\mathrm{a}, \mathrm{b}, *}$ \\ ${ }^{a}$ School of Physical and Occupational Therapy, McGill University, Montreal, Canada \\ ${ }^{\mathrm{b}}$ Feil and Oberfeld Research Center, Jewish Rehabilitation Hospital, Center for Interdisciplinary \\ Research in Rehabilitation of Greater Montreal, Laval, Canada \\ ${ }^{\mathrm{c}}$ Département de neurosciences, Université de Montréal, Montreal, Canada
}

\begin{abstract}
.
Purpose: Motor and cognitive impairments are common and often coexist in patients with stroke. Although evidence is emerging about specific relationships between cognitive deficits and upper-limb motor recovery, the practical implication of these relationships for rehabilitation is unclear. Using a structured review and meta-analyses, we examined the nature and strength of the associations between cognitive deficits and upper-limb motor recovery in studies of patients with stroke.

Methods: Motor recovery was defined using measures of upper limb motor impairment and/or activity limitations. Studies were included if they reported on at least one measure of cognitive function and one measure of upper limb motor impairment or function.

Results: Six studies met the selection criteria. There was a moderate association ( $r=0.43$; confidence interval; CI:0.09-0.68, $p=0.014$ ) between cognition and overall arm motor recovery. Separate meta-analyses showed a moderately strong association between executive function and motor recovery $(r=0.48$; CI:0.26-0.65; $p<0.001)$, a weak positive correlation between attention and motor recovery $(r=0.25$; CI:0.04- $0.45 ; p=0.023)$, and no correlation between memory and motor recovery $(r=0.42$; CI:0.16-0.79; $p=0.14$ ).

Conclusion: These results imply that information on the presence of cognitive deficits should be considered while planning interventions for clients in order to design more personalized interventions tailored to the individual for maximizing upper-limb recovery.
\end{abstract}

Keywords: Motor learning, upper limb, cognition, rehabilitation

\section{Introduction}

After stroke, approximately $60-80 \%$ of patients have upper limb motor deficits (Go et al., 2013; Langhorne et al., 2009) that can persist in 30-66\%

${ }^{*}$ Corresponding author: Mindy F. Levin, PhD, PT, School of Physical and Occupational Therapy, McGill University, 3630 Promenade Sir-William-Osler, Montreal, QC, H3G 1Y5, Canada. Tel.: +1 4506889550 (lab); Fax: +1 514398 6360; E-mail: mindy.levin@mcgill.ca. of cases into the chronic stage (Kwakkel et al., 1999). Upper limb recovery is important for minimizing longterm disability and improving the quality of life. Most rehabilitation interventions focus on facilitating recovery through motor learning principles (Kleim \& Jones, 2008). Motor learning involves perception, cognition and action processes described as the search for a task solution that emerges from an interaction of the individual with the desired action and the environment (Newell, 1991). 
Learning engages cognitive processes such as attention, memory and executive functioning, all of which may be affected by stroke (Hochstenbach et al., 1998). Research is increasingly focusing on the investigation of the impact of the manipulation of motor learning principles on upper limb motor improvement (Cirstea \& Levin, 2007). In studies of motor recovery, however, cognitive and motor elements have often been considered as separate systems. Indeed, most studies investigating the effectiveness of post-stroke upper limb rehabilitation interventions exclude individuals with stroke who have cognitive deficits. On the other hand, relationships between cognitive and motor deficits are increasingly being identified (Barker-Collo \& Feigin, 2006) at body structure and function (impairment), activity limitation and participation levels of the International Classification of Function (ICF; World Health Organization, n.d.). Cognitive dysfunction is recognized as influencing rehabilitation outcomes as well as predicting functional independence and participation after stroke (Paolucci et al., 1996; Heruti et al., 2002). For example, individuals with stroke who had more impaired cognition performed worse on the Functional Independence Measure (FIM) after rehabilitation compared to those with less cognitive impairment (Öneş et al., 2009). Rehabilitation outcomes identified using generic activity scales including the FIM and Barthel Index (BI), do not discriminate between use of the more affected vs. the less affected limbs nor do they specifically identify impairment and activity limitations such as endpoint performance variables and quality of movement of the affected limbs (Levin et al., 2009). Describing motor behavior at these two levels provides information about the movement of the endpoint (e.g., movement speed, precision and smoothness) and the movement patterns used to displace the endpoint (e.g. ranges of joint motion and/or coordination; Cirstea and Levin, 2007). Thus, generic scales are poor indicators of motor recovery of the upper limb at these levels.

Evidence is emerging about the specific relationship between cognitive deficits and upper limb improvement at both impairment and activity levels of the ICF. Information about these relationships is important to design personalized interventions more tailored to the individual in order to maximize upper limb recovery. The aim of this meta-analysis was to examine the evidence on the strength of the association between cognitive deficits and improvements in upper limb motor impairment and activity limitation in individuals with stroke.

\section{Methods}

\subsection{Search strategy}

A structured review of the English language literature covering five databases: Pubmed, Ovid MEDLINE, CINHAL, Embase, and ISI Web of Science was performed by AAM. Various combinations of MeSH terms and key words including; stroke, cerebrovascular accident, upper limb, cognit*, attention, memory and executive function were used. Reference lists of retrieved studies were searched to identify other pertinent articles.

\subsection{Selection criteria}

No date restrictions were imposed, yielding potential articles up to September 2014. Peer-reviewed articles reporting original research were included if 1) baseline cognition scores were provided; 2 ) arm motor impairment and/or activity limitation measures were included as study outcomes; 3 ) repetitive movement or more comprehensive motor rehabilitation interventions were delivered; 4) statistical associations between baseline cognition and motor outcome scores were done or could be derived from the data.

Studies were excluded if hemispatial neglect, a visuoperceptual disorder, agnosia or apraxia were included as cognitive deficits, since they are not due to purely cognitive etiologies (Greene, 2005; Hassa et al., 2011; Parton et al., 2004). Studies involving dual-task paradigms were excluded since improvement in dualtask performance after training could be due to changes in cognitive function without actual motor learning. Cross-sectional studies not investigating motor learning were also excluded, since it was not possible to identify changes due to motor learning from a single time-point measure. Furthermore, studies that measured improvement on motor subscales of generic functional tests (FIM and BI) were excluded since they are not specific to the upper limb and improved scores could reflect increased compensation with the less affected extremity (Levin et al., 2009) and/or improvement in lower limb ability.

\subsection{Study quality assessment}

All retrieved studies were reviewed by AAM and SKS and conflicts were resolved by MFL. Depending on the study design, studies were rated for soundness of methodology and reporting using valid and reliable 
quality of evidence scales: the 10-point PEDro Scale for Randomized Control Trials (RCT; Maher et al., 2003; Foley et al., 2006) and the 27-item Downs and Black Checklist for non-randomized studies (Downs \& Black, 1998). The 12-item Quality Assessment Tool for Before-After (Pre-Post) Studies with no Control Group (Jensen et al., 2014) was also used. Scores were normalized on each scale. Studies scoring $\geq 60 \%$, $40-59 \%$ or $<39 \%$ were rated as good, fair and poor respectively (Foley et al., 2006).

\subsection{Meta-analyses}

Meta-analyses (MedCalc, v14.8.1, Ostend, Belgium) examined strengths of associations between cognitive deficits and improvements in arm motor impairment and activity limitation. When an article reported more than one intervention, each intervention was entered as a separate study. For studies that used more than one cognitive measure and/motor outcome, the maximally correlated measures were used. Pooled association effects were calculated with weighted summary correlation coefficients (fixed effects model, $\mathrm{z}$ scores; Hedges \& Olkin, 1985). Heterogeneity was assessed using Cochran's Q and $\mathrm{I}^{2}$ for random effects (Dersimonian \& Laird, 1986). Studies were considered heterogeneous if $\mathrm{I}^{2}>50 \%$ (Higgins \& Green, 2011).

\section{Results}

\subsection{Search results}

A total of 202 articles were retrieved from Pubmed. Six articles were included, 5 of which met our selection criteria and one additional article was retrieved from the reference lists of the included articles. No additional articles were retrieved from the other databases (Fig. 1). Among the six included studies, there was one RCT (Cirstea et al., 2006), one non-randomized control trial (Skidmore et al., 2012), three pre-post studies (Boe et al., 2014; Barreca et al., 1999; Platz \& Denzler, 2002) and one cross-sectional study (Dancause et al., 2002), involving a total of 128 participants. Stroke chronicity varied from 3 weeks to 2.3 years (Table 1 ). The quality of the studies ranged from fair to good (Table 2).

\subsection{Measures of cognition}

All included studies used valid and reliable neuropsychological tests (Table 3). All but one study
(Barreca et al., 1999) examined two or more cognitive domains - attention, memory and executive function (information processing speed, planning abilities, cognitive flexibility) - via composite or individual domain scores (Table 3). One study (Barecca et al., 1999) examined executive function only. Studies used raw (Boe et al., 2014; Barreca et al., 1999), ranked (Dancause et al., 2002) or adjusted (Cirstea et al., 2006; Skidmore et al., 2012; Platz \& Denzler, 2002) scores for statistical analyses. The study samples were all heterogeneous in terms of severity of cognitive deficits except for one (Platz \& Denzler, 2002) that included a mildly affected stroke group. Seven of the ten subjects included in another study had average/above average executive function although they differed widely in attention scores (Dancause et al., 2002).

\subsection{Measures of arm motor improvement}

All studies used different clinical outcomes to measure baseline motor ability. One study (Platz \& Denzler, 2002) only included subjects with mild arm motor deficits, two only included subjects with mild/moderate arm deficits (Skidmore et al., 2012; Boe et al., 2014) while the other three had heterogeneous samples including subjects with more severe impairments (Cirstea et al., 2006; Barreca et al., 1999; Dancause et al., 2002).

Motor improvement occurred in participants of all studies (Table 3). Two studies (Cirstea et al., 2006; Dancause et al., 2002) assessed motor improvement using kinematically derived measures, while the others used only clinical measures to describe improvement (Table 1).

\subsection{Type of intervention}

Each study included different interventions or tasks. In two studies, participants practiced single tasks such as reaching or rapid elbow flexion (Cirstea et al., 2006; Dancause et al., 2002), and in the others, they participated in outpatient rehabilitation (Barreca et al., 1999), programs involving Arm Ability Training (AAT; Platz \& Denzler, 2002), repetitive task practice (Skidmore et al., 2012), or Constraint Induced Movement Therapy (CIMT; Boe et al., 2014) all of which likely involved multiple upper limb tasks. While principles of motor learning such as repetition were used in most studies (Cirstea et al., 2006; Dancause et al., 2002; Platz \& Denzler, 2002; Skidmore et al., 


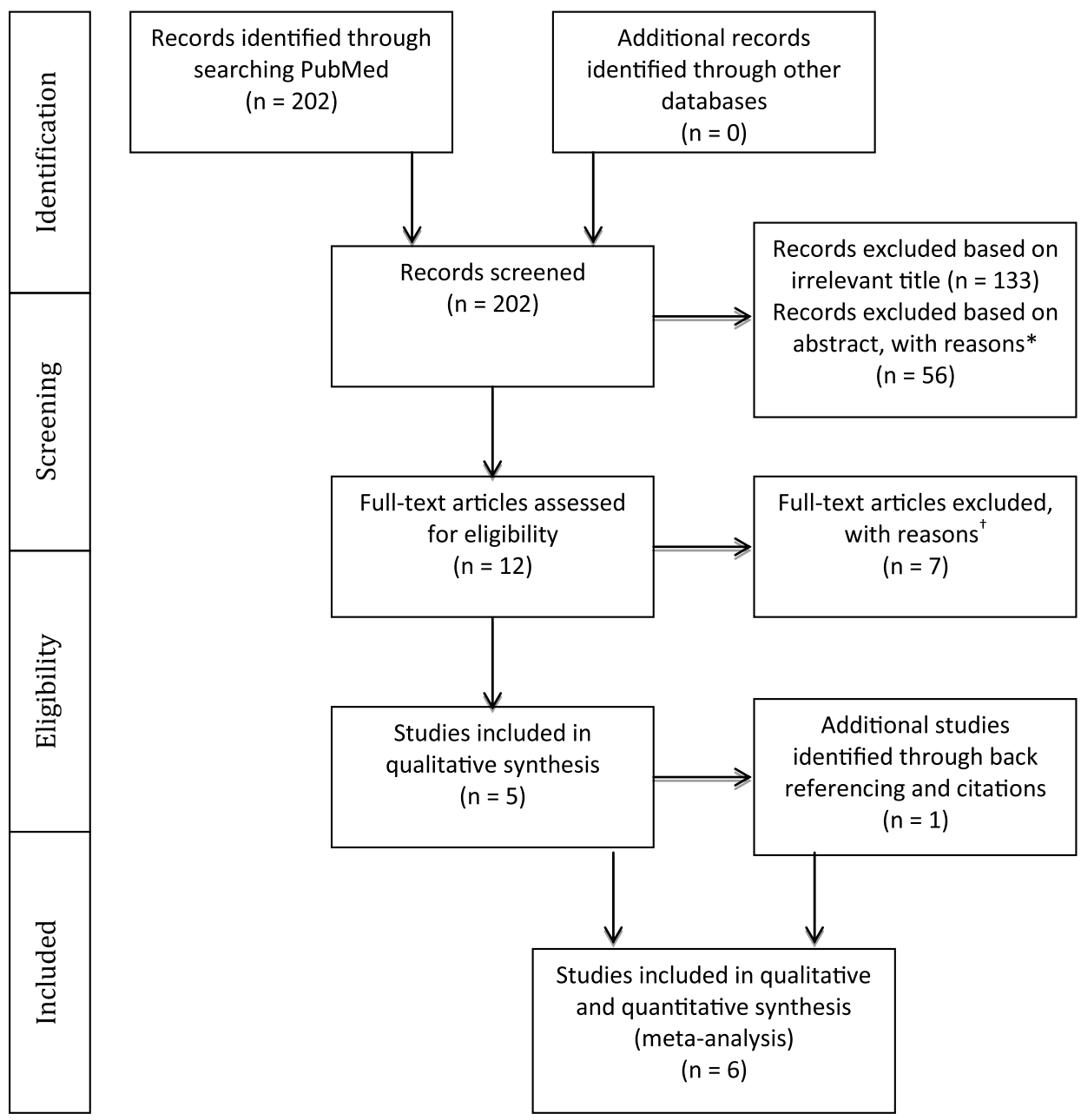

Fig. 1. PRISMA Flow Diagram. *Reasons for exclusion: exclusion of persons with impaired cognition $(n=20)$; no baseline cognitive assessment $(n=16)$; no upper limb motor outcome $(n=9)$; no repetitive movement or motor rehabilitation intervention provided $(n=5)$; Dual task interventions $(n=2)$; hemispatial neglect was the only cognitive predictor $(n=2)$; upper limb intervention review paper $(n=1)$; non-stroke study sample $(n=1){ }^{\dagger}$ Reasons for exclusion: statistical associations between baseline cognition and motor outcome scores were not done and could not be derived from the data provided $(n=5)$; no baseline cognitive assessment $(n=1)$; no motor intervention $(n=1)$.

2012; Boe et al., 2014), only two (Cirstea et al., 2006; Dancause et al., 2002) specified the type of feedback used and the manner in which it was delivered. The duration of the interventions varied widely among the studies from one day to 8 weeks and training intensity varied from 30 minutes to 6 hours per session (Table 1).

\subsection{Relationship between cognition and improvement of motor impairment}

Of the six studies, two (Barreca et al., 1999; Dancause et al., 2002) demonstrated a relationship between cognition and motor improvement, three found motor improvement to be independent of prestroke cognitive levels (Skidmore et al., 2012; Boe et al., 2014; Platz \& Denzler 2002) and one (Cirstea et al., 2006) showed a differential effect based on the type of intervention (Table 2). In order to determine the extent to which cognition and motor improvement are related, we performed a series of meta-analyses. The first analysis combined all studies irrespective of the cognitive domain assessed and the motor outcome used (Fig. 2, Table 4). A positive correlation of moderate strength was found between cognition and arm motor improvement ( $r=0.43$; CI: $0.09-0.68 ; p=0.014$ ). 
Table 1

Characteristics of studies included in the review

\begin{tabular}{|c|c|c|c|c|c|c|c|c|c|c|}
\hline \multirow[t]{2}{*}{$\begin{array}{l}\text { Study } \\
\text { name }\end{array}$} & \multirow[t]{2}{*}{ Design } & \multirow[t]{2}{*}{$\begin{array}{l}\mathrm{N}, \\
\text { Diagnosis }\end{array}$} & \multirow[t]{2}{*}{$\begin{array}{l}\text { Time since } \\
\text { stroke onset }\end{array}$} & \multicolumn{3}{|c|}{$\begin{array}{l}\text { Cognitive domains } \\
\text { tested }\end{array}$} & \multicolumn{2}{|c|}{$\begin{array}{c}\text { Motor outcome } \\
\text { Motor outcome measures }\end{array}$} & \multirow[t]{2}{*}{$\begin{array}{l}\text { Type of } \\
\text { intervention }\end{array}$} & \multirow[t]{2}{*}{$\begin{array}{l}\text { Assessment } \\
\text { time points }\end{array}$} \\
\hline & & & & $\overline{\mathrm{Att}}$ & Mem & $\begin{array}{l}\text { Exec } \\
\text { Funct }\end{array}$ & Kinematics & $\begin{array}{l}\text { Clinical } \\
\text { scales }\end{array}$ & & \\
\hline $\begin{array}{l}\text { Barreca et al. } \\
1999\end{array}$ & Pre-Post & $16 \mathrm{~S}$ & $3 \mathrm{wk}-1 \mathrm{yr}$ & $x$ & $x$ & $\checkmark$ & $x$ & UEFT & Outpatient rehabilitation & Admission, discharge \\
\hline $\begin{array}{l}\text { Dancause et al. } \\
2002\end{array}$ & $\begin{array}{l}\text { Cross- } \\
\text { sectional }\end{array}$ & $10 \mathrm{~S}$ & $6 \mathrm{mo}-2.3 \mathrm{yr}$ & $\checkmark$ & $\checkmark$ & $\checkmark$ & $\begin{array}{l}\text { UL kinematics } \\
\text { (endpoint, } \\
\text { elbow } \\
\text { movement) } \\
\text { Error correction } \\
\text { patterns }\end{array}$ & $\times$ & $\begin{array}{l}\text { One-trial learning } \\
\text { paradigm (correct } \\
\text { movement errors } \\
\text { occurring from sudden } \\
\text { introduction or removal } \\
\text { of load) }\end{array}$ & $\begin{array}{l}\text { Single day assessment } \\
\text { within each block of } \\
\text { trials }\end{array}$ \\
\hline $\begin{array}{l}\text { Platz \& Denzler } \\
\quad 2002\end{array}$ & Pre-Post & $25 \mathrm{~S}+7 \mathrm{TBI}$ & $3 \mathrm{wk}-6 \mathrm{mo}$ & $\checkmark$ & $\checkmark$ & $x$ & $\times$ & $\begin{array}{l}\text { TEMPA- time } \\
\text { taken to } \\
\text { complete tasks }\end{array}$ & Arm Ability Training & $\begin{array}{l}\text { Baseline, after 3-wk } \\
\text { intervention }\end{array}$ \\
\hline $\begin{array}{l}\text { Cirstea et al. } \\
2006\end{array}$ & RCT & $\begin{array}{c}35 \mathrm{~S} 3 \text { groups } \\
\mathrm{KP} n=14 \\
\mathrm{KR} n=14 \\
\mathrm{C} n=7\end{array}$ & $3-24 \mathrm{mo}$ & $\checkmark$ & $\checkmark$ & $\checkmark$ & $\begin{array}{l}\text { UL kinematics } \\
\text { (endpoint, } \\
\text { movement time, } \\
\text { velocity, } \\
\text { precision, } \\
\text { segmentation, } \\
\text { variability) }\end{array}$ & FM TEMPA & $\begin{array}{l}\text { Repetitive movement } \\
\text { practice (pointing } \\
\text { movement without } \\
\text { vision) using feedback } \\
\text { to promote learning }\end{array}$ & $\begin{array}{l}\text { Baseline, after 2-wk } \\
\text { intervention, 1-mo } \\
\text { follow-up }\end{array}$ \\
\hline $\begin{array}{l}\text { Skidmore et al. } \\
2012\end{array}$ & Non-RCT & $\begin{array}{l}20 \mathrm{~S} 2 \text { groups } \\
\text { CI } n=13 \\
\text { No CI } n=7\end{array}$ & $11 \pm 17 \mathrm{mo}$ & $\checkmark$ & $\checkmark$ & $\checkmark$ & $\times$ & ARAT & Repetitive task practice & $\begin{array}{l}\text { Baseline, after 4-wk } \\
\text { intervention, 24-wk } \\
\text { follow-up }\end{array}$ \\
\hline Boe et al. 2014 & Pre-Post & $21 \mathrm{~S}$ & 3-12 mo & $\checkmark$ & $\checkmark$ & $\checkmark$ & $x$ & WMFT & $\begin{array}{l}\text { Constraint Induced } \\
\text { Movement Therapy }\end{array}$ & $\begin{array}{l}\text { Baseline, after 2-wk } \\
\text { training, 3-mo } \\
\text { follow-up }\end{array}$ \\
\hline
\end{tabular}

Att- Attention; Mem-Memory; Exec Funct-Executive Function; RCT-Randomized Control Trial; S-stroke; C-healthy control; TBI-Traumatic Brain Injury; KP-Knowledge of performance; KR-Knowledge of results; CI-cognitive impairments; UL-upper limb; UEFT-Upper Extremity Function Test; TEMPA-Arm Function Test; FM-Fugl-Meyer (upper extremity subsacale);

ARAT-Action Research Arm Test; WMFT-Wolf Motor Function Test; $\checkmark$-Tested; ×-Not Tested. 
Table 2

Study quality determined by PEDro Scale, Downs and Black Checklist or Quality Assessment Tool for Before-After (Pre-Post) Studies with no Control Group based on percentage scoring $\geq 60 \%, 40-59 \%$ or $<39 \%$ (Good, Fair, Poor, respectively). The presence (YES) or absence (NO) of an association between cognitive and motor improvement scores is listed in column 4

\begin{tabular}{|c|c|c|c|}
\hline $\begin{array}{l}\text { Study } \\
\text { name }\end{array}$ & Score & $\begin{array}{l}\text { Quality of } \\
\text { study }\end{array}$ & $\begin{array}{l}\text { Association } \\
\text { present/not }\end{array}$ \\
\hline Barreca et al. 1999 & 7/11 (12) & Fair & Yes \\
\hline Dancause et al. 2002 & - & - & Yes \\
\hline Platz \& Denzler 2002 & $7 / 11(12)$ & Fair & No \\
\hline Cirstea et al. 2006 & $8 / 10$ & Good & KP group- Yes \\
\hline Skidmore et al. 2012 & $21 / 27$ & Good & $\begin{array}{c}\text { KR group- No } \\
\text { No }\end{array}$ \\
\hline Boe et al. 2014 & $8 / 11(12)$ & Good & No \\
\hline
\end{tabular}

Separate meta-analyses revealed a moderately strong association between executive function and motor improvement ( $r=0.48$; CI: $0.26-0.65$; $p<0.001$ ), a weak positive correlation between attention and motor improvement ( $r=0.25$; CI: 0.04-0.45; $p=0.023)$ and no correlation between memory and motor improvement $(r=0.42$; CI: $-0.16-0.79$; $p=0.14$; Fig. 3 , Table 5)

Additional meta-analyses showed stronger associations between cognition and motor improvement when kinematic motor outcomes are used rather than clinical scales $(r=0.72$; CI: $0.49-0.85 ; p<0.001$ and $r=0.13$, CI: $-0.21-0.45 ; p=0.45$ respectively, Table 6 ).

\section{Discussion}

The evidence on the strength of the association between cognitive deficits and improvements in upper limb motor impairment and activity limitations in patients who have sustained a stroke was examined. Our meta-analysis revealed, for the first time, a moderate association ( $r=0.43$; CI: $0.09-0.68)$ between cognition and overall arm motor improvement (Fig. 2) from a total of 6 moderate to high quality studies.

\subsection{Association between individual cognitive domains and arm motor improvement}

We found that deficits in executive functioning affect motor improvement to a greater extent than deficits in either attention or memory (Fig. 3, Table 5). This is consistent with findings from Levin et al. (2014), in which levels of executive functioning were more strongly correlated with learning arm movement patterns than other cognitive domains. Executive functions include task initiation, problem-solving, perseveration, abstract reasoning, planning, organization, mental flexibility and information processing (Lezak et al., 2012). Executive function is involved in appropriately modifying behaviour and adapting movement to changing environmental conditions when new information is available (Elliott, 2003). Thus, it may be more important than attention and memory for deriving benefit from therapies that use problem solving approaches such as CIMT (Boe et al., 2014), error correction through evaluation of results of a previous trial (Dancause et al., 2002) and adapting movement based on external cues (Cirstea et al., 2006).

Our results show a weak association between attention and improvements in arm impairments and activity limitations and no association between memory and motor improvement. This is in contrast to previous research demonstrating stronger associations between attention and improvement in arm activity scores (Carter et al., 1988; Robertson et al., 1997; Hyndman et al., 2008) and between memory and motor rehabilitation outcomes (Mount et al., 2007). Dissimilarities in the chronicity of the stroke population in previous studies and those included in the present meta-analysis, may account for the differences. Studies that found strong associations between attention/memory and motor improvement (Robertson et al., 1997; Hyndman et al., 2008; Mount et al., 2007) examined individuals with more acute stroke than those included in this review. The nature of the association between different cognitive domains and motor recovery may not be the same in the acute and chronic phases of stroke (Nys et al., 2005; Snaapshan \& de Leeuw, 2007; Barker-Collo et al., 2012). Indeed, Nys et al. (2005), Snaphaan and de Leeuw (2007) and Barker-Collo et al. (2012) found that while attention and memory impairments are common in the acute state after stroke, deficits in executive function are more prevalent in the chronic stage.

\subsection{Influence of severity of motor and cognitive deficits}

The severities of motor and cognitive deficits in individuals with stroke have previously been identified as strong predictors of motor recovery (Barker-Collo \& Feigin, 2006; Paolucci et al., 1996; Heruti et al., 2002). Our results may have been influenced by differences in the levels of cognitive and/or motor impairment of the participants between studies. For example, we found 
Table 3

Detailed methodology about cognitive and motor outcome measures used and results of studies included in the review

\begin{tabular}{|c|c|c|c|c|c|c|}
\hline Author & Chronicity & $\begin{array}{l}\text { Cognitive } \\
\text { measures }\end{array}$ & $\begin{array}{l}\text { Baseline } \\
\text { cognitive status }\end{array}$ & $\begin{array}{l}\text { Motor outcome } \\
\text { measures }\end{array}$ & $\begin{array}{l}\text { Baseline motor } \\
\text { status }\end{array}$ & Findings \\
\hline $\begin{array}{l}\text { Barreca } \\
\text { et al. } 1999\end{array}$ & $\begin{array}{l}\text { Hetero- } \\
\text { genous }\end{array}$ & $\begin{array}{l}\text {-Halstead Category Test } \\
\text { score assessing } \\
\text { executive functioning } \\
\text { (complex concept } \\
\text { formation and problem } \\
\text { solving abilities) }\end{array}$ & Decreased & $\begin{array}{l}\text { Clinical: } \\
\text {-Upper Extremity Function } \\
\text { Test (UEFT) score }\end{array}$ & $\begin{array}{l}\text { Severe: } \\
\text { Median CM stage on } \\
\text { admission: Arm = } 2 \\
\text { Hand=2 }\end{array}$ & $\begin{array}{l}\text { Mean UEFT change score }=14.9 \mathrm{pts} \\
\text { (range: } 0-63 \text { ) } \\
\text {-Initial Halstead category test scores } \\
\text { correlated with UEFT change scores } \\
(\mathrm{r}=-0.64 ; \mathrm{p}=0.005) \\
\text {-Initial UEFT and Halstead scores and } \\
\text { explained } 79.8 \% \text { of UEFT score at } \\
\text { discharge. } \\
\text {-High correlation between Halstead and CM } \\
\text { Arm (r }=-0.81) \text { and Hand ( } \mathrm{r}=-0.69) \text { scores } \\
\text { at discharge }\end{array}$ \\
\hline $\begin{array}{l}\text { Dancause } \\
\text { et al. } 2002\end{array}$ & Chronic & $\begin{array}{l}\text { Attention: } \\
\text {-Trail making tests A \& B } \\
\text {-ROCFT } \\
\quad \text { (visuospatial/non } \\
\text { verbal memory, } \\
\text { attention) } \\
\text { Memory: } \\
\text {-WMSS (verbal) } \\
\text {-ROCFT } \\
\text { Executive Function: } \\
\text {-WCST- (-mental } \\
\text { flexibility) } \\
\text {-Tower of London } \\
\text { (planning and problem } \\
\text { solving) } \\
\text {-Trail making test B } \\
\text { (attention, mental } \\
\text { flexibility) } \\
\text { Others: } \\
\text {-WAIS-R (abbrev. IQ) }\end{array}$ & $\begin{array}{l}\text { Mixed : } \\
\text { (7/10 had intact } \\
\text { trajectory, } \\
\text { executive } \\
\text { function) }\end{array}$ & $\begin{array}{l}\text { Kinematics: } \\
\text {-Performance (endpoint } \\
\text { velocity, position) } \\
\text {-Torque } \\
\text {-Error correction patterns } \\
\text { (\# trials required for } \\
\text { correction and \# errors } \\
\text { per block) }\end{array}$ & $\begin{array}{l}\text { Mixed: } \\
\text {-CM Arm - 2-6 } \\
\text {-FM 19-66 } \\
\text {-spasticity- } 4 \text { mild, } \\
6 \text { moderate to } \\
\text { severe }\end{array}$ & $\begin{array}{l}\text {-Movements in stroke slower than healthy } \\
\text { - Better memory and executive function } \\
\text { related to better adaptive behavior } \\
\text { and learning } \\
\text {-Combination of IQ, verbal, non verbal } \\
\text { memory and executive functioning } \\
\text { explained } 52.3 \% \text { of the variance in error } \\
\text { correction patterns } \\
\text {-Baseline FM motor scores and executive } \\
\text { function explained 100\% of the variance } \\
\text { in error correction behavior } \\
\text {-Executive functioning scores alone } \\
\text { accounted for 33\% of the variance }\end{array}$ \\
\hline
\end{tabular}


Table 3

(Continued)

\begin{tabular}{|c|c|c|c|c|c|c|}
\hline Author & Chronicity & $\begin{array}{l}\text { Cognitive } \\
\text { measures }\end{array}$ & $\begin{array}{l}\text { Baseline } \\
\text { cognitive status }\end{array}$ & $\begin{array}{l}\text { Motor outcome } \\
\text { measures }\end{array}$ & $\begin{array}{l}\text { Baseline motor } \\
\text { status }\end{array}$ & Findings \\
\hline $\begin{array}{l}\text { Platz \& } \\
\text { Denzler } \\
2002\end{array}$ & $\begin{array}{c}\text { Subacute } \\
\text { Stroke, } \\
\text { TBI }\end{array}$ & $\begin{array}{l}\text { Attention: } \\
\text { - Wiener } \\
\text { Reaktionsgera } \\
\text { (reaction time) } \\
\text {-Attention Battery } \\
\text { Test (visual } \\
\text { scanning and } \\
\text { perception) } \\
\text { Memory: } \\
\text { - Gollin } \\
\text { Fragmented } \\
\text { Picture Test } \\
\text { (learning) } \\
\text { Others: } \\
\text { - WAIS- R } \\
\text {-Hamilton Depression } \\
\text { Scale }\end{array}$ & Relatively intact & $\begin{array}{l}\text { Clinical: } \\
\text { - Arm Function Test } \\
\text { (TEMPA; time taken } \\
\text { to complete the } \\
\text { tasks) }\end{array}$ & $\begin{array}{l}\text { Mildly affected } \\
\text { shoulder abduction and elbow } \\
\text { flexion -Wrist/hand muscle } \\
\text { strength }>3 / 5 \text {, -Thumb-index } \\
\text { finger-opposition strength } \\
>2 / 5 \text { (MRC criteria), } \\
\text {-Selective finger movements and } \\
\text { precision grip preserved }\end{array}$ & $\begin{array}{l}\text {-Unilateral TEMPA time scores improved (mean } \\
\text { difference score } 14.6 ; \mathrm{t}=5.07, \mathrm{p}<0.001 \text { ) } \\
\text {-Total TEMPA time improved (mean difference } \\
\text { score } 34.7 ; \mathrm{t}=6.63, \mathrm{p}<0.001 \text { ). } \\
\text {-Large inter-individual variability (CV unilateral and } \\
\text { total TEMPA: } 113 \text { and } 87 \text {, respectively) } \\
\text { - Weak/no correlation between cognitive and motor } \\
\text { improvement scores }(\mathrm{r}=-0.25 \rightarrow 0.06) \\
\text {-Improvement in motor function was highly } \\
\text { correlated with degree of motor impairment } \\
\text { prior to training }(\mathrm{r}=0.84 \text { for unilateral TEMPA } \\
\text { tasks; } \mathrm{r}=0.50 \text { all TEMPA tasks)-despite mild } \\
\text { deficits }\end{array}$ \\
\hline $\begin{array}{r}\text { Cirstea } \\
\text { et al. } \\
2006\end{array}$ & Heterogenous & $\begin{array}{l}\text { Attention: } \\
\text {-ROCFT } \\
\text {-Stroop } \\
\text { Memory: } \\
\text {-WMSS } \\
\text {-RAVLT (verbal) } \\
\text {-ROCFT } \\
\text { (visuospatial) } \\
\text { Executive Function: } \\
\text {-WCST (mental } \\
\text { flexibility) } \\
\text {-Stroop (mental } \\
\text { flexibility) } \\
\text {-Tower of London } \\
\text { (planning and } \\
\text { problem solving) }\end{array}$ & Decreased & $\begin{array}{l}\text { Kinematics: } \\
\text { Performance } \\
\text {-endpoint } \\
\text { smoothness, } \\
\text { variability } \\
\text { precision, speed } \\
\text { Clinical: } \\
\text {-FM and TEMPA }\end{array}$ & $\begin{array}{l}\text { Mixed: } \\
\text {-able to reach with impaired } \\
\text { arm (at least stage } \\
2 \text { on CM Arm) }\end{array}$ & $\begin{array}{l}\text {-KP and KR groups } \\
\text { KR improved precision and movement speed } \\
\text { (less than KP) } \\
\text { - no correlation between precision and cognitive } \\
\text { scores } \\
\text { KP improved movement speed, segmentation and } \\
\text { variability without increasing precision) } \\
\text { - better retention (smoothness) related to better } \\
\text { verbal memory scores }\left(\mathrm{r}^{2}=0.95\right) \\
\text {-decreased variability related to better mental } \\
\text { flexibility/problem solving }\left(\mathrm{r}^{2}=0.94\right) \\
\text { - greater change in FM related to fewer deficits in } \\
\text { verbal and visuospatial memory and better } \\
\left.\text { planning ( } \mathrm{r}^{2}=0.96\right) \\
\text { - greater change in TEMPA related to better } \\
\text { planning }\left(\mathrm{r}^{2}=0.84\right) \\
\text { Control better retention of decreased speed } \\
\text { variability related to fewer deficits in mental } \\
\text { flexibility }\left(\mathrm{r}^{2}=0.83\right)\end{array}$ \\
\hline
\end{tabular}


Table 3

(Continued)

\begin{tabular}{|c|c|c|c|c|c|c|}
\hline Author & Chronicity & $\begin{array}{l}\text { Cognitive } \\
\text { measures }\end{array}$ & $\begin{array}{l}\text { Baseline } \\
\text { cognitive status }\end{array}$ & $\begin{array}{l}\text { Motor outcome } \\
\text { measures }\end{array}$ & $\begin{array}{l}\text { Baseline motor } \\
\text { status }\end{array}$ & Findings \\
\hline $\begin{array}{l}\text { Skidmore } \\
\text { et al. } \\
2012\end{array}$ & $\begin{array}{l}\text { Hetero- } \\
\text { genous }\end{array}$ & $\begin{array}{l}\text {-Repeatable Battery of } \\
\text { Neuropsychological Status } \\
\text { (immediate memory, attention, } \\
\text { visual spatial function, } \\
\text { language, and delayed } \\
\text { memory; age- adjusted } \\
\text { total index score) }\end{array}$ & Greatly decreased & $\begin{array}{l}\text { Clinical: } \\
\text {-Action Research } \\
\text { Arm Test (ARAT) }\end{array}$ & $\begin{array}{l}\text { Mild to moderate: } \\
\text {-Active movement at least } \\
15^{\circ} \text { elbow flexion, } 15^{\circ} \text { wrist } \\
\text { language, and delayed } \\
\text { extension, } 10^{\circ} \text { distal } \\
\text { interphalangeal flexion }\end{array}$ & $\begin{array}{l}\text {-Participants with and without cognitive } \\
\text { impairments improved significantly over time }\left(\mathrm{F}_{1,17}\right. \\
=84.48, \mathrm{p}<0.001) \text { regardless of cognitive status } \\
\quad\left(\mathrm{t}_{31}=1.42, \mathrm{p}=0.16\right) \text { or time since stroke }\left(\mathrm{t}_{17}=0.07 \text {, }\right. \\
\mathrm{p}=0.95) \text {. } \\
\text {-No association with initial cognition and degree of } \\
\text { improvement }(\mathrm{r}=-0.202)\end{array}$ \\
\hline $\begin{array}{l}\text { Boe } \\
\text { et al. } \\
2014\end{array}$ & Heterogenous & $\begin{array}{l}\text { Attention: } \\
\text {-Trail Making Tests A/B } \\
\text { Memory: } \\
\text { - RAVLT } \\
\text {-modified Location } \\
\text { Learning Test (learning) } \\
\text { Executive Function: } \\
\text {-Trail Making Test B } \\
\quad \text { (processing speed and } \\
\text { mental flexibility) } \\
\text {-Tower of London (planning } \\
\text { and problem solving) } \\
\text { Others: } \\
\text {-Boston Naming Test } \\
\text { (verbal ability) } \\
\text { - WAIS-III (Symbol Search, Digit } \\
\text { Span, Block Design: } \\
\text { processing speed, working } \\
\text { memory, visuospatial } \\
\text { construction respectively) }\end{array}$ & $\begin{array}{l}\text { Minimally } \\
\text { decreased }\end{array}$ & $\begin{array}{l}\text { Clinical: } \\
\text {-Wolf Motor } \\
\text { Function Test } \\
\text { (WMFT) }\end{array}$ & $\begin{array}{l}\text { Mild to moderate: } \\
\text {-at least } 10^{\circ} \text { active wrist } \\
\text { extension, thumb abduction/ } \\
\text { extension, and extension in } 2 \\
\text { other digits; } \\
\text {-ability to repeat these } \\
\text { movements } 3 \text { times in } 1 \\
\text { minute }\end{array}$ & $\begin{array}{l}\text {-Significant improvement in WMFT score with } \\
\text { intervention (mean change of } 6 \text { units; CI- 4.5-7.6; } \\
\text { p }<0.0001) \\
\text {-No significant correlation between cognition and } \\
\text { motor improvement at } 2 \text { wks post-test } \\
(\mathrm{r}=-0.18-0.21) \\
\text { - Processing speed, learning and memory, verbal } \\
\text { ability and executive function improved at } 3 \text { mo } \\
\text { follow-up but not attention and visuospatial } \\
\text { construction. }\end{array}$ \\
\hline
\end{tabular}

ROCFT- Rey-Osterrieth Complex Figure Test; WMSS- Wechsler Memory Scale Stories; WCST- Wisconsin Card Sorting Test; FM- Fugl-Meyer Assessment; CM- Chedoke McMaster; WAIS-R- Wechsler Adult Intelligence Scale-Revised; IQ- Intelligence Quotient; KR- knowledge of results; KP- knowledge of performance; Movt- movement; RAVLT- Rey Auditory Verbal Learning Test. ROCFT- Rey-Osterrieth Complex Figure Test. 


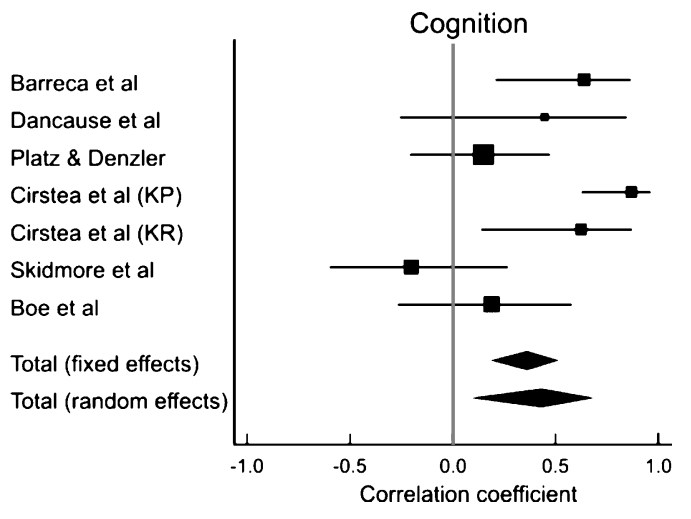

Fig. 2. Results of a meta-analysis examining the correlation between cognition and arm motor improvement. Larger squares represent larger study effect sizes. Diamonds indicate the pooled effects of results of individual studies. Diamond location indicates the estimated effect size and diamond width reflects the precision of the estimate.

that studies that included patients with more severe motor deficits, found stronger correlations (Cirstea et al., 2006; Barreca et al., 1999; Dancause et al., 2002) than those (Skidmore et al., 2012; Boe et al., 2014; Platz \& Denzler, 2002) that included participants with only mild to moderate motor deficits. We also found that two of the three studies (Boe et al., 2014; Platz \& Denzler, 2002) that identified no association between cognition and motor improvement included participants with only mild cognitive impairment. The third study (Skidmore et al., 2012) used a composite score to describe cognitive function. It is possible that different results may have been obtained if individual domain scores had been used. Therefore, our results are suggestive of different relationships between cognition and motor improvements depending upon the severity of motor and cognitive impairments.

\subsection{Impact of outcomes used to measure motor improvement}

In previous work examining associations between cognition and motor recovery, rather than characterizing motor recovery at the level of impairment, some studies assessed functional recovery using generic measures, such as motor subscales of the FIM or the BI. Results from these studies show weak (Fong et al., 2001) to moderately positive associations (Carter et al., 1988; Milinavičienè et al., 2011) between cognition and motor recovery similar to those obtained in our meta-analyses. However, generic outcomes are not specific to the recovery of motor activities of the more affected side of the body, do not discriminate between motor performance and quality of movement variables and combine improvements of the upper and lower limbs. Therefore, scores obtained from these measures are not helpful when designing targeted motor impairment or activity-based treatment interventions.

Motor improvement at the impairment or activity level can be assessed with both clinical and kinematic outcome measures. Our meta-analysis showed a stronger association between cognition and motor recovery when more sensitive kinematic measures were used (Table 6). Only two studies included in

Table 4

Results of the meta-analysis examining the correlation between cognition and arm motor improvement

\begin{tabular}{|c|c|c|c|c|c|}
\hline $\begin{array}{l}\text { Study } \\
\text { name }\end{array}$ & $\mathrm{n}$ & $\begin{array}{l}\text { Correlation } \\
\text { coefficient }\end{array}$ & $\begin{array}{c}95 \% \\
\mathrm{CI} \\
\end{array}$ & $\begin{array}{c}\mathrm{Z} \\
\text { score }\end{array}$ & $\begin{array}{c}p \\
\text { value }\end{array}$ \\
\hline Barreca et al. 1999 & 16 & 0.64 & $0.21-0.86$ & & \\
\hline Dancause et al. 2002 & 10 & 0.45 & $-0.25-0.84$ & & \\
\hline Platz \& Denzler 2002 & 33 & 0.15 & $-0.20-0.47$ & & \\
\hline Cirstea et al. 2006 (KP) & 14 & 0.87 & $0.63-0.96$ & & \\
\hline Cirstea et al. 2006 (KR) & 14 & 0.63 & $0.14-0.87$ & & \\
\hline Skidmore et al. 2012 & 20 & -0.20 & $-0.59-0.26$ & & \\
\hline Boe et al. 2014 & 21 & 0.19 & $-0.26-0.58$ & & \\
\hline Total (fixed effects) & 128 & 0.36 & $0.19-0.51$ & 3.92 & $<0.001$ \\
\hline Total (random effects) & 128 & 0.43 & $0.09-0.68$ & 2.46 & 0.014 \\
\hline \multicolumn{6}{|l|}{ Test for heterogeneity } \\
\hline $\mathrm{Q}$ & 21.31 & & & & \\
\hline $\mathrm{DF}$ & 6 & & & & \\
\hline Significance & $P=0.0016$ & & & & \\
\hline $\mathrm{I}^{2}$ & $71.86 \%$ & & & & \\
\hline
\end{tabular}

n-Sample size; CI-Confidence interval; Q-Cochran's Q; DF-degrees of freedom, $\mathrm{I}^{2}=100 \% \times(\mathrm{Q}-\mathrm{df}) / \mathrm{Q}$; KP-Knowledge of performance; KRKnowledge of results. 

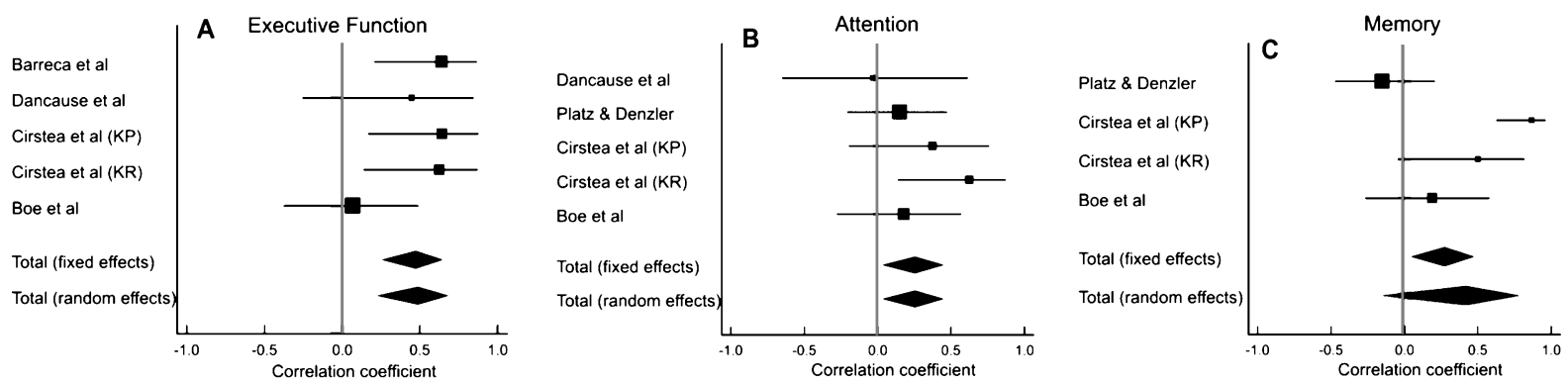

Fig. 3. Results of meta-analyses examining the correlation between arm motor improvement and (A) executive function, (B) attention and (C) memory. Larger squares represent larger study effect sizes. Diamonds indicate pooled effects of results of individual studies. Diamond location indicates the estimated effect size and diamond width reflects the precision of the estimate.

Table 5

Results of meta-analyses of studies that examined the correlation between arm motor improvement and (A) executive function, (B) attention and $(\mathrm{C})$ memory

\begin{tabular}{|c|c|c|c|c|c|}
\hline Study & $n$ & Correlation coefficient & $95 \% \mathrm{CI}$ & $\mathrm{z}$ & $p$ \\
\hline \multicolumn{6}{|l|}{ A. Executive Function } \\
\hline Barreca et al. 1999 & 16 & 0.64 & $0.21-0.86$ & & \\
\hline Dancause et al. 2002 & 10 & 0.45 & $-0.25-0.84$ & & \\
\hline Cirstea et al. (KP) 2006 & 14 & 0.64 & $0.17-0.88$ & & \\
\hline Cirstea et al. (KR) 2006 & 14 & 0.63 & $0.14-0.87$ & & \\
\hline Boe et al. 2014 & 21 & 0.07 & $-0.37-0.49$ & & \\
\hline Total (fixed effects) & 75 & 0.48 & $0.26-0.65$ & 3.996 & $<0.001$ \\
\hline Total (random effects) & 75 & 0.49 & $0.23-0.68$ & 3.469 & 0.001 \\
\hline \multicolumn{6}{|c|}{ Heterogeneity- $\mathrm{Q}=5.53 ; \mathrm{DF}=4 ; P=0.24 ; \mathrm{I}^{2}=27.67 \% ; 95 \% \mathrm{CI}$ for $\mathrm{I}^{2}=0-71.57$} \\
\hline \multicolumn{6}{|c|}{ B. Attention } \\
\hline Dancause et al. 2002 & 10 & -0.03 & $-0.65-0.61$ & & \\
\hline Platz and Denzler 2002 & 33 & 0.15 & $-0.20-0.47$ & & \\
\hline Cirstea et al. $2006(\mathrm{KP})$ & 14 & 0.38 & $-0.19-0.76$ & & \\
\hline Cirstea et al. 2006 (KR) & 14 & 0.63 & $0.14-0.87$ & & \\
\hline Boe et al. 2014 & 21 & 0.18 & $-0.27-0.57$ & & \\
\hline Total (fixed effects) & 92 & 0.25 & $0.037-0.45$ & 2.281 & 0.023 \\
\hline Total (random effects) & 92 & 0.25 & $0.037-0.45$ & 2.281 & 0.023 \\
\hline \multicolumn{6}{|c|}{ Heterogeneity- $\mathrm{Q}=3.72 ; \mathrm{DF}=4 ; P=0.45 ; \mathrm{I}^{2}=0 \% ; 95 \% \mathrm{CI}$ for $\mathrm{I}^{2}=0-78.94$} \\
\hline \multicolumn{6}{|c|}{ C. Memory } \\
\hline Platz and Denzler 2002 & 33 & -0.15 & $-0.47-0.20$ & & \\
\hline Cirstea et al. 2006 (KP) & 14 & 0.87 & $0.63-0.96$ & & \\
\hline Cirstea et al. 2006 (KR) & 14 & 0.50 & $-0.04-0.82$ & & \\
\hline Boe et al. 2014 & 21 & 0.19 & $-0.26-0.58$ & & \\
\hline Total (fixed effects) & 82 & 0.27 & $0.046-0.47$ & 2.343 & 0.019 \\
\hline Total (random effects) & 82 & 0.42 & $-0.16-0.79$ & 1.450 & 0.147 \\
\hline \multicolumn{6}{|c|}{ Heterogeneity- $\mathrm{Q}=18.62 ; \mathrm{DF}=3 ; P=0.0003 ; \mathrm{I}^{2}=83.89 \% ; 95 \%$ CI for $\mathrm{I}^{2}=59.36-93.62$} \\
\hline
\end{tabular}

n- Sample size; CI- Confidence interval; p- Level of significance; Q- Cochran's Q; DF- degrees of freedom, $\mathrm{I}^{2}=100 \% \times(\mathrm{Q}-\mathrm{df}) / \mathrm{Q} ; \mathrm{KP}-$ Knowledge of performance; KR- Knowledge of results.

our review (Cirstea et al., 2006; Dancause et al., 2002) examined the relationship between cognition and arm recovery using kinematic outcomes. Both studies found positive associations between motor performance and executive function (Cirstea et al., 2006; Dancause et al., 2002) and memory (Cirstea et al., 2006). Improvements in motor performance outcomes can indicate actual behavioural recovery or an increased use of compensatory movements (Levin et al., 2009). Movement quality outcomes were not measured in any of the studies included in our review. These outcomes have stronger associations with cognitive impairments (Levin et al., 2014) and are more sensitive to improvements in motor impairment than 
Table 6

Results of meta-analyses for association between cognition and arm motor improvement scores based on the motor outcome measures used

\begin{tabular}{|c|c|c|c|c|c|}
\hline Study & $n$ & Correlation coefficient & $95 \% \mathrm{CI}$ & $\mathrm{z}$ & $p$ \\
\hline \multicolumn{6}{|l|}{ A. Kinemetics } \\
\hline Dancause et al. 2002 & 10 & 0.45 & $-0.25-0.84$ & & \\
\hline Cirstea et al. $2006(\mathrm{KP})$ & 14 & 0.87 & $0.63-0.96$ & & \\
\hline Cirstea et al. 2006 (KR) & 14 & 0.63 & $0.14-0.87$ & & \\
\hline Total (fixed effects) & 38 & 0.72 & $0.49-0.85$ & 4.851 & $<0.001$ \\
\hline Total (random effects) & 38 & 0.71 & $0.37-0.88$ & 3.513 & $<0.001$ \\
\hline \multicolumn{6}{|c|}{ Heterogeneity- $\mathrm{Q}=3.58 ; \mathrm{DF}=2 ; P=0.17 ; \mathrm{I}^{2}=44.08 \% ; 95 \% \mathrm{CI}$ for $\mathrm{I}^{2}=0-83.30$} \\
\hline \multicolumn{6}{|l|}{ B. Clinical Scales } \\
\hline Barreca et al. 1999 & 16 & 0.64 & $0.21-0.86$ & & \\
\hline Platz and Denzler 2002 & 33 & 0.15 & $-0.20-0.47$ & & \\
\hline Cirstea et al. 2006 (KP) & 14 & 0.47 & $-0.08-0.80$ & & \\
\hline Cirstea et al. 2006 (KR) & 14 & -0.54 & $-0.83-0.02$ & & \\
\hline Skidmore et al. 2012 & 20 & -0.20 & $-0.59-0.26$ & & \\
\hline Boe et al. 2014 & 21 & 0.19 & $-0.26-0.58$ & & \\
\hline Total (fixed effects) & 118 & 0.13 & $-0.06-0.32$ & 1.335 & 0.182 \\
\hline Total (random effects) & 118 & 0.13 & $-0.21-0.45$ & 0.763 & 0.445 \\
\hline \multicolumn{6}{|c|}{ Heterogeneity- $\mathrm{Q}=14.71 ; \mathrm{DF}=5 ; P=0.01 ; \mathrm{I}^{2}=66.02 \% ; 95 \% \mathrm{CI}$ for $\mathrm{I}^{2}=18.69-85.80$} \\
\hline
\end{tabular}

n- Sample size; CI- Confidence interval; p- Level of significance; Q- Cochran's Q; DF- degrees of freedom; $\mathrm{I}^{2}=100 \% \times(\mathrm{Q}-\mathrm{df}) / \mathrm{Q}$; KPKnowledge of performance; KR- Knowledge of results.

clinical scales (Subramanian et al., 2010a). Therefore, it is suggested that kinematic outcomes should be included as motor impairment measures in future studies to obtain a better understanding of the relationship between motor recovery and cognitive deficits.

\subsection{Clinical implications}

Rehabilitation therapies for the upper limb in individuals who have sustained a stroke are designed to promote motor learning. It has been suggested that learning is enhanced by optimally challenging the individual through manipulation of the task difficulty according to the motor skill level of the performer, and their cognitive (information processing ability) capacity (Guadagnoli \& Lee, 2004). Learning is also related to performance or feedback-related intrinsic and extrinsic sensory information obtained during task performance (Chung et al., 2014; Subramanian et al., 2010b) which also depends on task complexity and cognitive processing. Our results suggest that clinicians should select appropriate motor learning approaches based on the characteristics of the individual and the specific motor outcomes to be improved. For example, one study (Cirstea et al., 2006) found no correlation between cognitive scores and motor improvement in a group of stroke subjects receiving KR feedback, but found a strong association between the two in a group receiving KP feedback. In the KP group, improvements in movement smoothness and precision were related to better memory, mental flexibility and planning abilities. It is possible that executive function and memory are more involved in processing information about moving and adapting movement behaviour. Thus, KP feedback should be prescribed with caution for individuals with specific cognitive deficits.

Cognitive impairments may affect the ability of the patient to understand and remember task instructions, plan and initiate self-directed activities and solve problems. Preliminary studies have suggested that individualizing treatment strategies based on individual cognitive and motor deficits may improve treatment effectiveness. For example, an RCT comparing the effectiveness of Neurodevelopmental Therapy (NDT, without cognitive rehabilitation) and Problem Oriented Willed Movement therapy (POWM, combined motor and cognitive rehabilitation) reported better motor outcomes in the POWM group compared to the NDT group (Tang et al., 2005).

\subsection{Future directions}

There is mounting interest in understanding the relationship between cognition and motor recovery. Evidence that treatment effectiveness may improve by integrating cognitive and motor rehabilitation is beginning to surface. However, additional informa- 
tion about optimal methods of treatment delivery is required to design more targeted therapies. For example, is discovery learning (trial and error learning) or guiding the patient towards a particular motor solution (errorless learning) more effective in individuals with stroke who have cognitive disorders? Evidence suggests that trial and error learning results in better skill retention than errorless learning approaches for individuals without memory deficits (Singer \& Pease, 1976), but errorless learning may be more effective for retention of information in people with cognitive disorders (O'Carroll et al., 1999; Wilson et al., 1994). Similarly, questions regarding the differential effects of timing and the mode of feedback delivery (visual/ tactile/ verbal) and efficacy of blocked versus random practice in patients with and without cognitive disorders are yet to be addressed.

\subsection{Limitations}

While this review provides important information about the interaction between cognitive and motor deficits in post-stroke individuals, results of the metaanalysis should be considered preliminary because of the small number of studies, as well as the small number of participants in each study. A limitation of the analysis is the lack of information about whether results may be related to lesion size and site.

\section{Conclusion}

Cognitive status affects therapy outcome but more research is needed to identify how severity of deficits, chronicity and intervention delivery impact this association. Further research in this area is essential in order to provide more personalized therapies.

\section{Sources of funding}

AAM and SKS are supported by fellowships of the Heart and Stroke Foundation of Canada - Focus on Stroke program; MFL holds a Canada Research Chair in Motor Recovery and Rehabilitation.

\section{Disclosures}

None.

\section{Conflict of interest}

The authors declare that there is no conflict of interest.

\section{References}

Barker-Collo, S., \& Feigin, V. (2006). The impact of neuropsychological deficits on functional stroke outcomes. Neuropsychol Rev, 16(2), 53-64.

Barker-Collo, S., Starkey, N., Lawes, C.M., Feigin, V., Senior, H., \& Parag, V. (2012). Neuropsychological profiles of 5-year ischemic stroke survivors by Oxfordshire stroke classification and hemisphere of lesion. Stroke, 43(1), 50-55.

Barreca, S.R., Finlayson, A.M., Gowland, C.A., \& Basmajian, J.V. (1999). Use of the Halstead Category Test as a cognitive predictor of functional recovery in the hemiplegic upper limb: A cross-validation study. Clin Neuropsychol, 13(2), 171-181.

Boe, E.W., Pedersen, A.D., Pedersen, A.R., Nielsen, J.F., \& Blicher, J.U. (2014). Cognitive status does not predict motor gain from post stroke constraint-induced movement therapy. NeuroRehabilitation, 34(1), 201-207.

Carter, L.T., Oliveira, D.O., Duponte, J., \& Lynch, S.V. (1988). The relationship of cognitive skills performance to activities of daily living in stroke patients. Am J Occup Ther, 42(7), 449-455.

Chung, E., Lee, B.H., \& Hwang, S. (2014). Core stabilization exercise with real-time feedback for chronic hemiparetic stroke: A pilot randomized controlled trials. Restor Neurol Neurosci, 32(2), 313-321.

Cirstea, C.M., Ptito, A., \& Levin, M.F. (2006). Feedback and cognition in arm motor skill reacquisition after stroke. Stroke, 37(5), $1237-1242$.

Cirstea, M.C., \& Levin, M.F. (2007). Improvement of arm movement patterns and endpoint control depends on type of feedback during practice in stroke survivors. Neurorehabil Neural Repair, 21(5), 398-411.

Dancause, N., Ptito, A., \& Levin, M.F. (2002). Error correction strategies for motor behavior after unilateral brain damage: Short-term motor learning processes. Neuropsychologia, 40(8), 1313-1323.

Dersimonian, R., \& Laird, N. (1986). Meta-analysis in clinical trials. Control Clin Trials, 7(3), 177-188.

Downs, S.H., \& Black, N. (1998). The feasibility of creating a checklist for the assessment of the methodological quality both of randomised and non-randomised studies of health care interventions. J Epidemiol Community Health, 52(6), 377-384.

Elliott, R. (2003). Executive functions and their disorders. Br Med Bull, 65(1), 49-59.

Foley, N.C., Bhogal, S.K., Teasell, R.W., Bureau, Y., \& Speechley, M.R. (2006). Estimates of quality and reliability with the physiotherapy evidence-based database scale to assess the methodology of randomized controlled trials of pharmacological and nonpharmacological interventions. Phys Ther, 86(6), 817-824.

Fong, K.N., Chan, C.C., \& Au, D.K. (2001). Relationship of motor and cognitive abilities to functional performance in stroke rehabilitation. Brain Inj, 15(5), 443-453. 
Go, A.S., Mozaffarian, D., Roger, V.L., Benjamin, E.J., Berry, J.D., Borden, W.B., Turner, M.B., et al. (2013). Heart disease and stroke statistics-2013 update: A report from the American Heart Association. Circulation, 127(1), e6-e245.

Greene, J.D. (2005). Apraxia, agnosias, and higher visual function abnormalities. J Neurol Neurosurg Psychiatry, 76(Suppl 5), v25-v34.

Guadagnoli, M.A., \& Lee, T.D. (2004). Challenge point: A framework for conceptualizing the effects of various practice conditions in motor learning. J Mot Behav, 36(2), 212-224.

Hassa, T., Schoenfeld, M.A., Dettmers, C., Stoppel, C.M., Weiller, C., \& Lange, R. (2011). Neural correlates of somatosensory processing in patients with neglect. Restor Neurol Neurosci, 29(4), 253-263.

Hedges, L.V., \& Olkin, I. (1985). Statistical Methods for Metaanalysis. Orlando: Academic Press.

Heruti, R.J., Lusky, A., Dankner, R., Ring, H., Dolgopiat, M., Barell, V., Adunsky, A., et al. (2002). Rehabilitation outcome of elderly patients after a first stroke: Effect of cognitive status at admission on the functional outcome. Arch Phys Med Rehabil, 83(6), 742-749.

Higgins, J.P., \& Green, S. (2011). Cochrane Handbook for Systematic Reviews of Interventions. Chichester: John Wiley \& Sons.

Hochstenbach, J., Mulder, T., van Limbeek, J., Donders, R., \& Schoonderwaldt, H. (1998). Cognitive decline following stroke: A comprehensive study of cognitive decline following stroke. J Clin Exp Neuropsychol, 20(4), 503-517.

Hyndman, D., Pickering, R.M., \& Ashburn, A. (2008). The influence of attention deficits on functional recovery post stroke during the first 12 months after discharge from hospital. J Neurol Neurosurg Psychiatry, 79(6), 656-663.

International Classification of Functioning, Disability and Health (ICF). Geneva, Switzerland: WHO; 2002. World Health Organization web site. http://www.Who.Int/classifications/icf/ training/icfbeginnersguide.Pdf. Accessed November 25 2014.

Jensen, M.D., Ryan, D.H., Donato, K.A., Apovian, C.M., Ard, J.D., Comuzzie, A.G., Yanovski, S. Z., et al. (2014). Guidelines (2013) for managing overweight and obesity in adults. Obesity, 22(S2), S1-S410.

Kleim, J.A., \& Jones, T.A. (2008). Principles of experiencedependent neural plasticity: Implications for rehabilitation after brain damage. J Speech Lang Hear Res, 51(1), S225-S239.

Kwakkel, G., Wagenaar, R.C., Twisk, J.W., Lankhorst, G.J., \& Koetsier, J.C. (1999). Intensity of leg and arm training after primary middle-cerebral-artery stroke: A randomised trial. Lancet, 354(9174), 191-196.

Langhorne, P., Coupar, F., \& Pollock, A. (2009). Motor recovery after stroke: A systematic review. Lancet Neurol, 8(8), 741-754

Levin, M.F., Kleim, J.A., \& Wolf, S.L. (2009). What do motor "recovery" and "compensation" mean in patients following stroke? Neurorehabil Neural Repair, 23(4), 313-319.

Levin, M.F., Subramanian, S.K., Chilingaryan, G., \& Sveistrup, H. (2014). Influence of depression and cognitive deficits on use of feedback for upper limb recovery in chronic stroke. Neurorehabil Neural Repair, 28(9), 924.
Lezak, M.D., Howieson, D.B., Bigler, E.D., \& Tranel, D. (2012). Neuropsychological Assessment. New York: Oxford University Press

Maher, C.G., Sherrington, C., Herbert, R.D., Moseley, A.M., \& Elkins, M. (2003). Reliability of the PEDro scale for rating quality of randomized controlled trials. Phys Ther, 83(8), 713-721.

Milinavičienė, E., Rastenyte, D., \& Kriščiūnas, A. (2011). Effectiveness of the Second-Stage Rehabilitation in Stroke Patients With Cognitive Impairment. Medicina (Kaunas), 47(9), 486-493.

Mount, J., Pierce, S.R., Parker, J., DiEgidio, R., Woessner, R., \& Spiegel, L. (2007). Trial and error versus errorless learning of functional skills in patients with acute stroke. NeuroRehabilitation, 22(2), 123-132.

Newell, K.M. (1991). Motor skill acquisition. Ann Rev Psychol, 42(1), 213-237.

Nys, G.M., Van Zandvoort, M.J., De Kort, P.L., Jansen, B.P., Van der Worp, H.B., Kappelle, L.J., \& De Haan, E.H. (2005). Domainspecific cognitive recovery after first-ever stroke: A follow-up study of 111 cases. J Int Neuropsychol Soc, 11(07), 795-806.

O’Carroll, R.E., Russell, H.H., Lawrie, S.M., \& Johnstone, E.C. (1999). Errorless learning and the cognitive rehabilitation of memory-impaired schizophrenic patients. Psychol Med, 29(01), 105-112.

Öneş, K., Yalçinkaya, E.Y., Toklu, BÇ, \& Cağlar, N. (2009). Effects of age, gender, and cognitive, functional and motor status on functional outcomes of stroke rehabilitation. NeuroRehabilitation, 25(4), 241-249.

Paolucci, S., Antonucci, G., Gialloreti, L.E., Traballesi, M., Lubich, S., Pratesi, L., \& Palombi, L. (1996). Predicting stroke inpatient rehabilitation outcome: The prominent role of neuropsychological disorders. Eur Neurol, 36(6), 385-390.

Parton, A., Malhotra, P., \& Husain, M. (2004). Hemispatial Neglect. J Neurol Neurosurg Psychiatry, 75(1), 13-21.

Platz, T., \& Denzler, P. (2002). Do psychological variables modify motor recovery among patients with mild arm paresis after stroke or traumatic brain injury who receive the Arm Ability Training? Restor Neurol Neurosci, 20(1), 37-49.

Robertson, I.H., Ridgeway, V., Greenfield, E., \& Parr, A. (1997). Motor recovery after stroke depends on intact sustained attention: A 2-year follow-up study. Neuropsychology, 11(2), 290-295.

Singer, R.N., \& Pease, D. (1976). A comparison of discovery learning and guided instructional strategies on motor skill learning, retention, and transfer. Res $Q, 47(4), 788-796$.

Skidmore, E.R., Becker, J.T., Whyte, E.M., Huber, L.M., Waterstram, L.F., Ward, A.A., \& Holm, M.B. (2012). Cognitive Impairments and Depressive Symptoms Did Not Impede Upper Extremity Recovery in a Clinical Repetitive Task Practice Program after Stroke: A Pilot Study. Am J Phys Med Rehabil, 91(4), 327-336.

Snaphaan, L., \& de Leeuw, F.E. (2007). Poststroke memory function in nondemented patients: A systematic review on frequency and neuroimaging correlates. Stroke, 38(1), 198-203. 
Subramanian, S.K., Yamanaka, J., Chilingaryan, G., \& Levin, M.F. (2010a). Validity of movement pattern kinematics as measures of arm motor impairment poststroke. Stroke, 41(10), 23032308.

Subramanian, S.K., Massie, C.L., Malcolm, M.P., \& Levin, M.F. (2010b). Does provision of extrinsic feedback result in improved motor learning in the upper limb poststroke? A systematic review of the evidence. Neurorehabil Neural Repair, 24(2), 113-124.
Tang, Q.P., Yang, Q.D., Wu, Y.H., Wang, G.Q., Huang, Z.L., Liu, Z.J., Fan, Z.Y. (2005). Effects of problem-oriented willed-movement therapy on motor abilities for people with poststroke cognitive deficits. Phys Ther, 85(10), 1020-1033.

Wilson, B.A., Baddeley, A., Evans, J., \& Shiel, A. (1994). Errorless learning in the rehabilitation of memory impaired people. Neuropsychol Rehabil, 4(3), 307-326. 